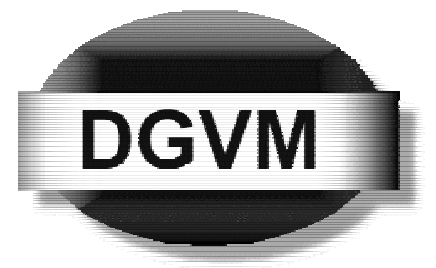

\section{Deutsche Gesellschaft für Verhaltensmedizin und Verhaltensmodifikation (DGVM)}

Prof. Dr. Ulrike Ehlert (Schriftführerin)

Psychologisches Institut der Universität Zürich

Klinische Psychologie II, Zürichbergstr. 43

CH-8044 Zürich

Tel. +41 1 634-30 97, Fax -36 96

E-mail schriftfuehrerin@dgvm-online.de,www.dgvm-online.de

\section{Bericht über den achten Internationalen Kongress der Verhaltensmedizin, 25.-28. August 2004 in Mainz}

Mehr als 800 Wissenschaftler und Praktiker aus 54 Ländern nahmen am achten Internationalen Kongress der Verhaltensmedizin im wunderschönen Kurfürstlichen Schloss in Mainz teil. Während der vier Kongresstage konnten die Teilnehmer aus über 850 wissenschaftlichen Beiträgen und zahlreichen Workshops ihr individuelles Programm zusammenstellen. Gemäß dem Ziel des Kongresses, Sozialwissenschaften, Verhaltensforschung, Medizin und Gesundheitsförderung thematisch und praktisch zu integrieren, zeichneten sich die Beiträge durch eine große thematische Vielfalt aus. Neueste Forschungsergebnisse zu verhaltensmedizinischen Aspekten der kardiovaskulären Erkrankungen, HIV, Asthma, Krebserkrankungen und Diabetes ebenso wie zu körperlicher Aktivität, Gesundheitsförderung, Abhängigkeitserkrankungen, chronischem Schmerz und vielen anderen Themen wurden in diversen Vorträgen und Symposien vorgestellt und kritisch diskutiert. Das Programm, das der Vorsitzende des Komitees, Prof. Dr. Neil Schneiderman, sowie Prof. Dr. Joost Dekker, Prof. Dr. Winfried Rief und weitere Mitarbeiter zusammengestellt hatten, ist durch die Kongressteilnehmer sehr positiv beurteilt worden.

International bekannte Experten für Verhaltensmedizin, Dr. Sheldon Cohen, Dr. Herta Flor und Dr. George Kaplan, gewährten im Rahmen ihrer «keynote lectures» interessante Einblicke in ihre bisherige Forschungsarbeit. Weitere Gastvorlesungen hielten Dr. Mike Antoni, Dr. Clemens Kirsch- baum, Dr. Johannes Siegrist, Dr. Jane Wardle und Dr. Anuar Zaini.

Hier nur einige Eindrücke aus den zahlreichen interessanten Vorträgen: Frau Dr. Flors Vortrag fasste bisherige Ergebnisse zur Plastizität des Gehirns insbesondere bei Schmerzerkrankungen zusammen und eröffnete Perspektiven für weitere Forschungsfelder (z.B. bei chronischem Tinnitus) in diesem Bereich. Dr. Cohen stellte die letzten 20 Jahre seiner Forschung zu psychosozialen Einflussfaktoren der Erkältung vor. Mit seinen zahlreichen Experimenten, in denen Personen unter verschiedenen Bedingungen Viren ausgesetzt werden, die Erkältungen oder Grippe hervorrufen können, konnte Dr. Cohen Zusammenhänge zwischen externen Faktoren wie kritischen Lebensereignissen, biologischen Faktoren wie hormonellen Veränderungen, behavioralen Faktoren wie Rauchen und der Anfälligkeit für Erkältungskrankheiten nachweisen. Dr. Mike Antoni evaluierte ein kognitiv-behaviorales Stressbewältigungsprogramm bei 300 Frauen mit Brustkrebs. Die Intervention konnte depressive Symptome, intrusive Kognitionen und Immunsystemparameter positiv beeinflussen. Psychologische Stressbewältigung, so die Schlussfolgerung, hat einen Einfluss auf die Krankheit selbst, zusätzlich zur bereits vielfach nachgewiesenen Erhöhung der Lebensqualität der Krebspatienten. Besonders eindrucksvoll war während des gesamten Kongresses der Dialog zwischen führenden Forschern und Nachwuchswissenschaftlern und -wissenschaftlerinnen verschiedener Disziplinen aus vielen Ländern und Kulturen. So entstand z.B. im Symposium zum Thema «Physische Aktivität und Rehabilitation bei Störungen im Muskel-Skelett-Bereich: psychologische Perspektiven» unter der Leitung von Prof. Dr. Basler ein reger Austausch zwischen schwedischen, deutschen und australischen Wissenschaftlerinnen zur Regelung der psychologischen Ausbildungsinhalte bei der Physiotherapeutenschulung in den jeweiligen Ländern. In einem anderen Symposium erläuterte ein Forscher aus Surinam (Südamerika) den Zuhörern zunächst die geografische Lage und kulturellen Besonderheiten seines Landes, ehe er auf die interessanten Forschungsergebnisse zur Gesundheitsförderung bei Altenheimbewohnern in seiner Heimat einging. Sehr erfreulich war die Teilnahme von Wissenschaftlern und Wissenschafterinnen aus Osteuropa, z.B. Russland, Ungarn und Rumänien, sowie aus arabischen Ländern, z.B. Iran. Der praktischen Seite der Verhaltensmedizin wurde in den zahlreichen Workshops, zum Beispiel zu «Biofeedback bei somatoformen Störungen» oder «Motivational Interviewing» gedient. Dank dem Mainzer Organisationskomitee unter Leitung von Prof. Dr. Hiller gelang ein reibungsloser Verlauf des

\begin{tabular}{ll}
\hline KARGER & $\odot$ 2004 S. Karger GmbH, Freiburg \\
$\begin{array}{l}\text { Fax +49761 45207 14 } \\
\begin{array}{l}\text { E-mail Information@Karger.de } \\
\text { www.karger.com }\end{array}\end{array}$ & $\begin{array}{l}\text { Accessible online at: } \\
\text { www.karger.com/ver }\end{array}$ \\
&
\end{tabular}


Kongresses, versüßt durch die einmalige Atmosphäre des Kurfürstlichen Schlosses und der Mainzer Altstadt. Während des prunkvollen Banketts wurde reichlich gegessen und bis spät in die Nacht getanzt. Anlässlich des bevorstehenden neunten Internationalen Kongresses der Verhaltensmedizin in Bangkok im Jahre 2006 veranstalteten die thailändischen Kollegen eine unvergessliche Abschlussfeier mit thailändischem Essen, Musik und einmaliger Gastfreundlichkeit. Dank der lehrreichen und spannenden Kongresstage und der Warmherzigkeit der zukünftigen Gastgeber sind insbesondere junge Forscher und Forscherinnen motiviert worden, den bevorstehenden Kongress durch viele neue Forschungsergebnisse zur Verhaltensmedizin zu bereichern.

Dipl.-Psych. Julia Glombiewski, Universität Marburg

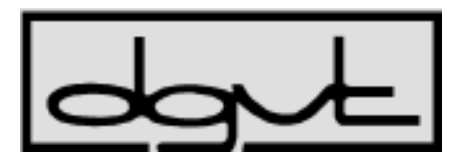

\section{Deutsche Gesellschaft für Verhaltenstherapie e.V. (DGVT)}

Bundesgeschäftsstelle

Postfach 1343

D-72003 Tübingen

Tel. 07071/943494, Fax 943435

E-maildgvt@dgvt.de,www.dgvt.de

\section{World Mental Health Day, 10. Oktober 2004: «Körperliche und seelische Gesundheit gehören zusammen"}

Der jährlich stattfindende World Mental Health Day stellte in diesem Jahr den nachhaltigen und ganzheitlichen Gesundheitsbegriff in den Mittelpunkt. In über 150 Ländern haben Mitgliedsorganisationen der World Federation for Mental Health (WFMH) - wie die DGVT - durch Informationen und verschiedenste Aktionen auf den Zusammenhang zwischen körperlicher und seelischer Gesundheit hingewiesen. Ausgangspunkt und Hintergrund des diesjährigen Mottos waren aktuelle Forschungsergebnisse, die den engen Zusammenhang zwischen psychischen und physischen Erkrankungen erneut belegen. Diese Erkenntnisse müssen gerade angesichts der notwendigen Reformen der psychosozialen Versorgungssysteme stärker beachtet werden - sowohl bei vorbeugenden Gesundheitsprogrammen wie auch im Praxis- und Klinikalltag. So erleben beispielsweise Menschen mit chronischen Erkrankungen häufig emotionale und psychische Störungen, die nicht angemessen erkannt und behandelt werden. Alle, die im Gesundheitssektor arbeiten, benötigen das Wissen, die Fertigkeiten und die Bereitschaft, mit seelisch beeinträchtigten Menschen zu arbeiten, Anzeichen psychischer Erkrankungen rechtzeitig zu erkennen sowie die emotionale und seelische Befindlichkeit des Patienten bei der medizinischen Behandlung einer «körperlichen» Erkrankung niemals außer Acht zu lassen. Dies gilt vor allem für die Primärversorgung durch niedergelassene Ärzte, aber auch für ambulante Dienste im nichtmedizinischen Sektor. Sie müssen dafür qualifiziert werden, psychische Anteile körperlicher Erkrankungen besser zu erkennen, um die PatientInnen angemessen zu behandeln. Ein niederschwelliger Zugang und der Erhalt des Erstzugangs zu einem Psychotherapeuten sind daher unabdingbar. Und: Im Zusammenhang mit dem geplanten Präventionsgesetz ist die Politik gefordert, gerade der Vermeidung psychischer Erkrankungen besonders Platz einzuräumen, da diese in einem engen Zusammenhang zu körperlichen Erkrankungen stehen. Die Beteiligung von PsychotherapeutInnen ist daher zwingend erforderlich.

\section{Inhouse-Schulung in Verhaltenstherapie und Verhaltensmodifikation im Kinder- und Jugendheim Linzgau}

Die Leitung des Linzgau Kinder- und Jugendheims in Überlingen trat Ende 2003 mit dem Wunsch an die DGVT heran, ihre MitarbeiterInnen kompakt und gemäß deren Bedürfnissen in ihrer konkreten Arbeit in Verhaltenstherapie und Verhaltensmodifikation (VT/VM) zu schulen. Hintergrund dieser Anfrage war, dass die DGVT bereits seit Jahren Fortbildungen in VT/VM anbietet und auch bereits Erfahrungen im einem vergleichbaren Kontext sammeln konnte.

Im Rahmen des ersten Treffens in der Einrichtung ging es einerseits um das gegenseitige Kennenlernen und andererseits um die Präzisierung der Aufgabenstellung, um eine genau auf den Bedarf der Einrichtung angepasste Inhouse-Weiterbildung zu entwickeln. Dabei wurde deutlich, dass in dieser Einrichtung - insbesondere aufgrund der engen Zusammenarbeit mit einer verhaltenstherapeutisch orientierten Kinder- und Jugendlichenpsychiaterin und einzelner bereits durchgeführter Fortbildungen zu verschiedenen Störungsbildern - bereits (verhaltens)therapeutische Kenntnisse vorhanden waren, an die angeknüpft werden konnte, die aber auch integriert werden wollten und sollten. Eine besondere Herausforderung bestand darin, dass in dieser Einrichtung ganz unterschiedliche Berufsgruppen in sehr verschiedenen Aufgabenfeldern arbeiten. Daraus resultierten nicht nur ungleiche Voraussetzungen für eine gemeinsame Fortbildung, sondern auch mannigfaltige Erwartungen an das Abstraktionsniveau und die praktischen Anwendungsmöglichkeiten der zu vermittelnden Inhalte. Aus dem Orientierungsgespräch resultierte folgendes Ergebnis: Es wurden drei jeweils eintägige, Fortbildungsveranstal- 
tungen konzipiert, in denen ein Überblick über die Entwicklung der Verhaltenstherapie bis heute gegeben wurde und eine Darstellung und ein erstes Erfahren von verhaltenstherapeutischen Standardmethoden. Gleichzeitig sollten auf diesem Wege mögliche, noch bestehende Vorbehalte gegenüber den Verfahren der VM abgebaut werden, um eine möglichst gute Compliance zu erreichen. Das Fortbildungsangebot stieß in der Einrichtung bereits bei der Vorankündigung auf sehr großes Interesse, so dass sich die ursprünglich geplante TeilnehmerInnenzahl von ca. 20 auf 35 erhöhte. Die Evaluation der Fortbildung zeigte, dass deren Erwartungen erfüllt wurden. Die genau auf den Bedarf abgestimmte Inhouse-Fortbildung hat sich als ein sehr guter Ansatz erwiesen, verhaltenstherapeutisches und verhaltensmodifikatorisches Know-how in diesem Bereich der Kinder- und Jugendhilfe zu vermitteln.

\section{Ausbildung in Psychologischer Psychotherapie und Kinder- und Jugendlichenpsychotherapie: Geplante Lehrgangsstarts}

Lehrgänge in Kinder- und Jugendlichenpsychotherapie beginnen im Frühjahr 2005 in Krefeld (Januar 2005), Dresden, Hannover/Hildesheim (April/Mai 2005), München/Bad Tölz und Bad Schussenried.

Lehrgänge in Psychologischer Psychotherapie beginnen im Frühjahr 2005 in Bonn, Hannover/Hildesheim und Münster. Ende Oktober hat in Bad Schussenried ein Lehrgang begonnen, in dem noch Plätze frei sind.

Anmeldungen zur Ausbildung nach dem Psychotherapeutengesetz sind - unabhängig von den genannten Lehrgangsstarts in der zweiten Hälfte diesen Jahres - kontinuierlich für alle regionalen Ausbildungszentren möglich.
Die Zeitschrift erscheint vierteljährlich; pro Jahr erscheint 1 Band $\mathrm{zu}$ je 4 Heften. Bezugspreise für Jahrgang 14, 2004: Print-Abonnement EUR 125,-, Online-Abonnement EUR 125,-, Kombi-Abonnement Print/Online EUR 158,-, einschließlich MwSt., zuzüglich Postgebühren. Der Abonnementpreis ist im Voraus zahlbar. Das Abonnement der Zeitschrift läuft weiter, wenn es nicht spätestens 4 Wochen vor Abschluss eines Bandes abbestellt wird. Abonnementbestellungen können bei jeder Buchhandlung oder direkt beim Verlag aufgegeben werden:

\section{Deutschland:}

S. Karger Verlag für Medizin und

Naturwissenschaften $\mathrm{GmbH}$

Lörracher Str. 16a

D-79115 Freiburg

Tel. +49761452070

Fax +497614520714

E-mail Information@Karger.de

\section{Übrige Länder:}

S. Karger AG

Allschwilerstr. 10

Postfach

CH-4009 Basel

Tel. +416130611 11

Fax +41613061234

E-mail Karger@Karger.ch
Anzeigen:

S. Karger Verlag für Medizin und Naturwissenschaften $\mathrm{GmbH}$

Lörracher Str. 16 a

D-79115 Freiburg

Tel. +49761452070

Gültig ist die Preisliste Nr. 9 vom 1. Januar 2004.

Für den Inhalt außerhalb des redaktionellen Teiles (insbesondere Anzeigen, Industrieinformationen, Pressezitate und Kongressinformationen) übernehmen Schriftleitung, Beirat und Verlag keine Gewähr. Eine Markenbezeichnung kann warenzeichenrechtlich geschützt sein, auch wenn bei ihrer Verwendung in dieser Zeitschrift das Zeichen ${ }^{\circledR}$ oder ein anderer Hinweis auf etwa bestehende Schutzrechte fehlen sollte. Für Satzfehler, insbesondere bei Dosierungsangaben, wird keine Gewähr übernommen. Die Zeitschrift sowie alle in ihr enthaltenen einzelnen Beiträge und Abbildungen sind urheberrechtlich geschützt. Jede Verwertung, die nicht ausdrücklich vom Urheberrechtsgesetz zugelassen ist, bedarf der vorherigen Zustimmung des Verlags. Das gilt insbesondere für Vervielfältigungen, Bearbeitungen, Übersetzungen, Mikroverfilmungen und die Einspeicherung und Verarbeitung in elektronischen Systemen.

\section{(c) Copyright 2004 by S. Karger}

Verlag für Medizin und Naturwissenschaften $\mathrm{GmbH}$

Lörracher Str. 16a

D-79115 Freiburg

Verlagsleitung und presserechtlich verantwortlich: Sibylle Hopf Produktionsleitung: Georg Brunner

Anzeigenleitung: Susanne Meister

Gesamtherstellung:

KONKORDIA GmbH, Bühl

Das Medienunternehmen

Bibliographische Dienste

Current Contents/Social \& Behavioral Sciences

Research Alert

Social Science Citation Index

PsycINFO

Reference Update 